\title{
Substitutions between dairy products and risk of stroke: results from the European Investigation into Cancer and Nutrition-Netherlands (EPIC-NL) cohort
}

\author{
Anne Sofie D. Laursen ${ }^{1 *}$, Ivonne Sluijs ${ }^{2}$, Jolanda M. A. Boer ${ }^{3}$, W. M. Monique Verschuren ${ }^{2,3}$, \\ Yvonne T. van der Schouw ${ }^{2}$ and Marianne U. Jakobsen ${ }^{1,4}$ \\ ${ }^{1}$ Research Unit for Epidemiology, Department of Public Health, Aarhus University, 8000 Aarhus, Denmark \\ ${ }^{2} J u l i u s$ Center for Health Sciences and Primary Care, University Medical Center Utrecht, Utrecht University, 3584 Utrecht, \\ The Netherlands \\ ${ }^{3}$ Centre for Nutrition, Prevention and Health Services, National Institute for Public Health and the Environment (RIVM), \\ 3720 Bilthoven, The Netherlands \\ ${ }^{4}$ Division for Diet, Disease Prevention and Toxicology, National Food Institute, Technical University of Denmark, \\ 2800 Kongens Lyngby, Denmark \\ (Submitted 9 May 2018 - Final revision received 17 January 2019 - Accepted 22 February 2019 - First published online 19 June 2019)
}

\section{Abstract}

The association between intake of different dairy products and the risk of stroke remains unclear. We therefore investigated substitutions between dairy product subgroups and risk of stroke. We included 36886 Dutch men and women. Information about dairy product intake was collected through a FFQ. Dairy products were grouped as low-fat milk, whole-fat milk, buttermilk, low-fat yogurt, whole-fat yogurt, cheese and butter. Incident stroke cases were identified in national registers. We used Cox proportional hazards regression to calculate associations for substitutions between dairy products with the rate of stroke. During a median follow-up of $15 \cdot 2$ years we identified 884 stroke cases ( 503 ischaemic and 244 haemorrhagic). Median intake of total dairy products was four servings/d. Low-fat yogurt substituted for whole-fat yogurt was associated with a higher rate of ischaemic stroke (hazard ratio $(\mathrm{HR})=2.58(95 \% \mathrm{CI} 1 \cdot 11,5.97) /$ serving per d). Whole-fat yogurt as a substitution for any other subgroup was associated with a lower rate of ischaemic stroke (HR between 0.33 and $0.36 /$ serving per $\mathrm{d}$ ). We did not observe any associations for haemorrhagic stroke. In conclusion, whole-fat yogurt as a substitution for low-fat yogurt, cheese, butter, buttermilk or milk, regardless of fat content, was associated with a lower rate of ischaemic stroke.

Key words: Dairy products: Fermented milk: Cheese: Butter: Stroke: Substitution models: Cohort studies

The Dietary Approaches to Stop Hypertension (DASH) diet ${ }^{(1)}$ is recommended for stroke prevention ${ }^{(2)}$. The diet prescribes a high daily intake of low-fat dairy products, while restricting the intake of regular-fat or high-fat dairy products. However, a study that compared a modified DASH diet, including wholefat dairy products and no low-fat dairy products, with the original DASH diet observed a similar beneficial effect on blood pressure as with the original DASH $\operatorname{diet}^{(3)}$. Also, the modified DASH diet was found to reduce plasma TAG and very-low-density lipoprotein cholesterol concentrations without increasing low-density lipoprotein cholesterol ${ }^{(3)}$, a potentially beneficial improvement in lipid profile. Most studies on dairy products and the risk of stroke have investigated intakes of total dairy products, total milk or dairy products categorised as either low-fat or whole-fat ${ }^{(4-8)}$. However, dairy products comprise a range of individual product types with individual nutritional properties ${ }^{(9)}$, and therefore these categorisations may be too broad. For instance, in a recent meta-analysis of eighteen follow-up studies investigating dairy product intake and the risk of stroke, it was observed that the intake of total whole-fat dairy products was associated with a lower risk of stroke, while the intake of whole-fat milk was associated with a higher risk of stroke ${ }^{(10)}$. Moreover, the intake of total fermented dairy products, including cheese, was associated with a marginally statistically non-significant lower risk of stroke $^{(10)}$. The authors thus call for future research with detailed information about product type and fat content. The studies

Abbreviations: DASH, Dietary Approaches to Stop Hypertension; DHD2015, Dutch Healthy Diet index 2015; EPIC-NL, European Investigation into Cancer and Nutrition-Netherlands; HR, hazard ratio.

* Corresponding author: Anne Sofie D. Laursen, email asdl@ph.au.dk 
included in this meta-analysis all investigated differences in the intake of total dairy products or dairy product subgroups while adjusting for total energy intake, thus holding it constant. Once energy intake is held constant, individuals with different intakes of dairy products will also differ in other non-specified energyproviding foods. Hence, these studies do not address whether one type of dairy product is to be preferred over another. Using specified food substitution analyses allows for a direct comparison between different types of dairy products. Only one previous study has investigated substitutions between dairy products and the risk of stroke, and observed that whole-fat yogurt products as a substitution for low-fat yogurt products, cheese or milk, regardless of fat content, was associated with a lower rate of stroke ${ }^{(11)}$, but these results have yet to be confirmed by other studies.

Our aim was to investigate the associations for substitutions between dairy product subgroups with incident stroke in a Dutch cohort that habitually consume large quantities and varieties of dairy products.

\section{Methods \\ Study population}

We used data from the Dutch participants of the European Investigation into Cancer and Nutrition (EPIC-NL), which has previously been described ${ }^{(12)}$. The EPIC-NL cohort comprised two cohorts - Prospect and MORGEN (Monitoring Project on Risk Factors for Chronic Diseases). The Prospect study included 17357 women aged 49-70 years, and the MORGEN study included 22654 men and women aged 21-64 years. All participants were recruited from 1993 to 1997, and the protocols for the cohorts were designed in collaboration, yielding compatible infrastructure. The studies complied with the Declaration of Helsinki and were approved by local medical ethical committees. All participants gave informed consent to participate in the study ${ }^{(12)}$.

Of the 40011 recruited participants, we excluded 1763 participants, who did not give permission to register linkage for vital status or disease occurrence or for whom the cause of death was missing, and another 453 participants, who reported having had a stroke prior to enrolment. Furthermore, we excluded participants in the top and bottom $0.5 \%$ of the ratio of energy intake to estimated basal metabolic rate ( $n$ 352) and those with missing exposure or covariate information ( $n 557$ ), yielding a study sample of 36886 men and women (online Supplementary Fig. S1).

\section{Dietary assessment}

Information about food intake was obtained through a semiquantitative FFQ with seventy-nine main items covering habitual consumption of 178 foods. Intake of dairy products was specifically addressed in items regarding breakfast cereals; butter and cheese eaten on bread; milk and yogurt for drinking; dairy products used in coffee; butter used for cooking; and dairy-based desserts.

The reproducibility and validity of the questionnaire has been investigated $^{(13)}$. The Spearman rank correlation coefficients for the validity assessed by comparison with twelve 24-h diet recalls were 0.64 and 0.38 for cheese among men and women, respectively, and 0.71 and 0.79 for milk and milk products among men and women, respectively.

For the present study we divided dairy product intake into the following groups: (1) low-fat milk (skimmed and semi-skimmed milk, $<2 \%$ fat), (2) whole-fat milk (whole-fat, raw and powdered milk, $\geq 3 \%$ fat), (3) buttermilk, (4) low-fat yogurt products (skimmed and semi-skimmed regular and drink yogurt, $<2 \%$ fat, curd), (5) whole-fat yogurt products (regular and drink yogurt, $\geq 3 \%$ fat), (6) cheese and (7) butter. The dairy product intake was expressed in servings/d and in $\mathrm{kcal} / \mathrm{d}$. For milk and yogurt products the serving size was $200 \mathrm{~g}$; and for cheese and butter it was 20 and $6 \mathrm{~g}$, respectively. We did not include the intake of custard, whipped cream and chocolate milk in the analyses.

\section{Stroke ascertainment}

Stroke cases were defined according to the International Classification of Diseases, 10th Edition, as codes I60-I66. Data on stroke occurrence were obtained through a standardised register for hospital discharge diagnoses administered by the Dutch Centre for Health Care Information since 1990. The register was linked to the cohort participants on the basis of birth date, sex, postal code and general practitioner by a validated probabilistic method as previously described ${ }^{(12,14)}$. Information about vital status was collected via municipal registries. Subsequently, primary and secondary causes of death were obtained through linkage with data from Statistics Netherlands. The stroke cases were classified as ischaemic or haemorrhagic. Participants were followed until the date of an incident stroke, death from another cause, loss to follow-up or were censored at 31 December 2010, whichever came first.

\section{Covariate information}

Baseline characteristics were collected using a self-administered questionnaire except for blood pressure and anthropometrical measurements, which were collected at a physical examination.

Educational attainment was categorised as low (primary up to intermediate vocational education), intermediate (intermediate vocational education and higher secondary education) or high (higher vocational education and university). BMI was calculated as weight divided by height-squared $\left(\mathrm{kg} / \mathrm{m}^{2}\right)$. Physical activity level was defined as inactive, moderately inactive, moderately active or active, according to the Cambridge Physical Activity Index ${ }^{(7,12,15)}$. Hypertension was defined on the basis of either self-reported physician-diagnosed hypertension, selfreported use of antihypertensive medication, systolic blood pressure $\geq 140 \mathrm{mmHg}$ or diastolic blood pressure $\geq 90 \mathrm{mmHg}$ at the baseline examination. Hypercholesterolaemia and previous myocardial infarction were self-reported. Prevalent diabetes mellitus was collected through self-report and linkage with hospital discharge diagnosis registers and was verified by the general practitioner ${ }^{(16)}$. Information about alcohol intake and intake of food groups other than dairy foods was collected with the FFQ. We also calculated a modified version of the Dutch Healthy Diet index 2015 (DHD2015) in order to adjust for overall diet quality ${ }^{(17)}$. The index measures adhered to the 2015 dietary guidelines from the Dutch Health Council ${ }^{(18)}$. With the data available, we were able to include thirteen of the fifteen original indicators: fruit, vegetables, wholegrain products, legumes, nuts, 
dairy products, fish, tea, fat and oils, red meat, processed meat, sweetened beverages and fruit juices and alcohol. The dairy product component, however, was excluded because dairy products are our main exposure.

\section{Statistical analyses}

The associations for substitutions between subgroups of dairy products and the rate of total, ischaemic and haemorrhagic stroke were investigated using Cox proportional hazard regression with age as the underlying time scale and adjusted for cohort (as stratum variable).

The substitution model (model 1) included the intake of all individual subgroups of dairy products (servings/d) except the dairy product subgroup to be substituted (i.e. six out of seven subgroups were included in the model) and a variable representing the total number of servings of dairy products and total energy intake $(\mathrm{kcal} / \mathrm{d})$. It follows that the hazard ratio (HR) for each dairy product subgroup in the model can be interpreted as the difference in the rate of stroke for one serving/d higher intake of the subgroups included in the model and a concomitant lower intake of the subgroup left out of the model. Using the same analytical approach, we also analysed isoenergetic substitutions of $100 \mathrm{kcal} / \mathrm{d}(418 \mathrm{~kJ} / \mathrm{d})$. The substitution analyses were adjusted as follows: education (categorical), BMI-adjusted waist circumference (residuals of waist circumference regressed on BMI) (continuous), smoking (categorical), physical activity (categorical), alcohol intake (5-knot spline) (model 2) and DHD2015 (continuous) (model 3). We further adjusted the analyses for the potential intermediate conditions such as hypertension, hypercholesterolaemia, diabetes mellitus and myocardial infarction at baseline in a separate model (model 4).

The assumption of independent delayed entry was investigated by including the date of enrolment in the models. As no association between the date of enrolment and stroke was found, the assumption was deemed satisfied. The proportional hazards assumption tested with Schoenfeld residuals was satisfied. In order to determine departures from linearity in the substitution models, we plotted martingale residuals against the dairy product subgroup variables and included a LOWESS smooth and found no departure from linearity.

We further investigated potential effect modification between dairy product variables and the covariates sex, age, BMI-adjusted waist circumference, smoking, alcohol, physical activity and DHD2015. We used likelihood ratio tests where we compared models (model 3) with and without interaction terms for the respective dairy product and covariate variables. We found no indication of effect modification ( $P$ values between 0.07 and 0.99).

We performed sensitivity analyses where participants diagnosed with hypertension, hypercholesterolaemia, diabetes mellitus or myocardial infarction at baseline were excluded, as the treatment for these conditions include dietary changes. These analyses were only performed for total and ischaemic stroke due to limited cases of haemorrhagic stroke. All analyses were performed using Stata 13.1 (StataCorp).

\section{Results}

During a median follow-up of $15 \cdot 2$ years we identified 884 stroke cases, including 503 ischaemic, 244 haemorrhagic and
137 unclassified types. Participant characteristics across quintiles of dairy product subgroups are given in Table 1 and online Supplementary Tables S1-S6. While there were no pronounced differences in characteristics between quintiles of low-fat milk intake (Table 1), participants with a high intake of whole-fat milk or butter were more likely to be men, current smokers and have a higher alcohol intake (online Supplementary Tables S1 and S6). For both low-fat and whole-fat yogurt, those with high intakes compared with the lowest were more likely to be women and drink less alcohol and less likely to be current smokers or physically inactive. Moreover, participants with a high intake of yogurt had higher intakes of fruit and vegetables and lower intakes of red and processed meat compared with participants with the lowest intake (online Supplementary Tables S3 and S4).

For ischaemic stroke, we did not observe an association for low-fat milk as a substitution for whole-fat milk ( $\mathrm{HR}=0.97$ (95\% CI $0.73,1.30)$ ), whereas low-fat yogurt products as a substitution for whole-fat yogurt products were associated with a higher rate $(\mathrm{HR}=2.58$ (95\% CI 1.11, 5.97)). Also, whole-fat yogurt products as a substitution for milk, buttermilk, cheese or butter were associated with a lower rate of ischaemic stroke (low-fat milk: HR $=0.34$ (95\% CI 0.15, 0.75); whole-fat milk: $\mathrm{HR}=0.33(95 \%$ CI $0.14,0.74)$; buttermilk: HR $=0.35(95 \%$ CI 0.16, 0.78); cheese: HR $=0.35$ (95\% CI 0.16, 0.77); butter: $\mathrm{HR}=0.36(95 \%$ CI $0 \cdot 16,0 \cdot 79)$ ) (Table 2$)$. We did not observe any association for substitutions between dairy product subgroups with the rate of total or haemorrhagic stroke (online Supplementary Table S7 and Table 3).

When we repeated the analyses without the participants who reported a history of hypertension, hypercholesterolaemia, diabetes mellitus or myocardial infarction at baseline, the patterns of associations remained the same, although the results did not reach statistical significance (online Supplementary Tables S8 and S9).

When isoenergetic substitutions of $100 \mathrm{kcal} / \mathrm{d}(418 \mathrm{~kJ} / \mathrm{d})$ were performed, we observed similar patterns of associations as for substitutions of servings (online Supplementary Tables S10-S12).

\section{Discussion}

In this prospective cohort study we observed that the intake of whole-fat yogurt products as a substitution for all other dairy product subgroups was associated with a lower rate of ischaemic stroke. We did not observe any associations for substitutions between dairy products and the rate of total or haemorrhagic stroke.

Our study has important strengths but also limitations. We had detailed information about the intake of several types of dairy products, which enabled us to directly compare individual diary product subgroups. We collected data on dairy product intake using a validated $\mathrm{FFQ}^{(12)}$. The correlation coefficients for the validity assessed by comparison with twelve 24-h diet recalls were 0.64 and 0.38 for men and women, respectively, for cheese and 0.71 and 0.79 for men and women, respectively, for milk and milk products, indicating that some non-differential misclassification of dairy product intake may be present. In the validation study, milk and yogurt were grouped together, and therefore, we do not know the validity for individual types of milk and yogurt. Although an FFQ is a suitable instrument to 
NS British Journal of Nutrition

Table 1. Participant characteristics in the European Investigation into Cancer and Nutrition-Netherlands (EPIC-NL) cohort ( $n 36886$ ) (Medians and $80 \%$ central ranges; numbers of participants; percentages)

\begin{tabular}{|c|c|c|c|c|c|c|c|c|c|c|c|c|}
\hline & & & \multicolumn{10}{|c|}{ Quintiles of low-fat milk } \\
\hline & \multicolumn{2}{|c|}{ All participants } & \multicolumn{2}{|r|}{ Q1 } & \multicolumn{2}{|r|}{ Q2 } & \multicolumn{2}{|c|}{ Q3 } & \multicolumn{2}{|r|}{ Q4 } & \multicolumn{2}{|r|}{ Q5 } \\
\hline & Median & $80 \%$ range & Median & $80 \%$ range & Median & $80 \%$ range & Median & $80 \%$ range & Median & $80 \%$ range & Median & $80 \%$ range \\
\hline Total stroke $(n)$ & \multirow{2}{*}{\multicolumn{2}{|c|}{$\begin{array}{c}884 \\
25 \cdot 4\end{array}$}} & \multicolumn{2}{|r|}{181} & \multirow{2}{*}{\multicolumn{2}{|c|}{$\begin{array}{l}171 \\
26 \cdot 2\end{array}$}} & \multirow{2}{*}{\multicolumn{2}{|c|}{$\begin{array}{r}161 \\
30 \cdot 0\end{array}$}} & \multirow{2}{*}{\multicolumn{2}{|c|}{$\begin{array}{l}201 \\
22.6\end{array}$}} & \multicolumn{2}{|r|}{170} \\
\hline Sex, men (\%) & & & \multicolumn{2}{|r|}{20.5} & & & & & & & \multicolumn{2}{|r|}{27.5} \\
\hline Age (years) & 51.4 & $31 \cdot 1-63 \cdot 4$ & $52 \cdot 2$ & $35 \cdot 2-63.3$ & 51.3 & $32 \cdot 3-63 \cdot 2$ & $50 \cdot 2$ & $28 \cdot 7-62 \cdot 5$ & $52 \cdot 3$ & $32 \cdot 2-64 \cdot 8$ & $50 \cdot 8$ & $27 \cdot 8-63 \cdot 2$ \\
\hline BMI $\left(\mathrm{kg} / \mathrm{m}^{2}\right)$ & $25 \cdot 1$ & $22 \cdot 9-30 \cdot 8$ & $25 \cdot 1$ & $21 \cdot 1-31 \cdot 3$ & $25 \cdot 1$ & $21 \cdot 1-30 \cdot 6$ & 24.9 & $21 \cdot 1-30 \cdot 3$ & $25 \cdot 3$ & $21 \cdot 4-30 \cdot 9$ & $25 \cdot 2$ & $21 \cdot 3-30 \cdot 9$ \\
\hline \multicolumn{13}{|l|}{ Waist circumference (cm) } \\
\hline Men & $92 \cdot 0$ & $79 \cdot 0-106 \cdot 3$ & 93.0 & $80 \cdot 0-107 \cdot 8$ & $92 \cdot 0$ & $79 \cdot 8-106 \cdot 5$ & 91.5 & $78 \cdot 5-105 \cdot 0$ & 91.4 & $78 \cdot 5-106 \cdot 0$ & $92 \cdot 0$ & $79 \cdot 0-106 \cdot 8$ \\
\hline Women & 81.5 & $70 \cdot 2-97 \cdot 0$ & $81 \cdot 3$ & $70 \cdot 0-97 \cdot 6$ & $81 \cdot 0$ & $70 \cdot 0-96 \cdot 0$ & $80 \cdot 5$ & 70.0-95.7 & $82 \cdot 0$ & $71 \cdot 0-97 \cdot 0$ & $82 \cdot 0$ & $71 \cdot 0-97 \cdot 5$ \\
\hline Low educational attainment (\%) & \multicolumn{2}{|r|}{57.9} & \multicolumn{2}{|r|}{$58 \cdot 3$} & \multicolumn{2}{|r|}{$58 \cdot 0$} & \multicolumn{2}{|r|}{57.6} & \multicolumn{2}{|r|}{$60 \cdot 0$} & \multicolumn{2}{|r|}{55.4} \\
\hline Current smoker (\%) & \multirow{2}{*}{\multicolumn{2}{|c|}{$\begin{array}{l}30 \cdot 3 \\
7.4\end{array}$}} & & $30 \cdot 8$ & & $30 \cdot 5$ & & 33.6 & & 27.4 & & $29 \cdot 3$ \\
\hline Physically inactive (\%) & & & & $9 \cdot 0$ & & 7.9 & & $7 \cdot 3$ & & $6 \cdot 6$ & & $6 \cdot 2$ \\
\hline Alcohol consumption (g/d) & $5 \cdot 0$ & $0 \cdot 0-30 \cdot 3$ & $6 \cdot 8$ & $0.01-34.6$ & $4 \cdot 8$ & $0.02-30.1$ & $5 \cdot 0$ & $0.02-29.7$ & 4.4 & $0.02-28.7$ & 4.4 & $0.01-28 \cdot 1$ \\
\hline Hypertension (\%) & & $37 \cdot 2$ & & $40 \cdot 6$ & & $36 \cdot 5$ & & 33.7 & & 39.0 & & $36 \cdot 3$ \\
\hline Hypercholesterolaemia (\%) & & 8.5 & & $9 \cdot 4$ & & 8.4 & & 7.4 & & $8 \cdot 3$ & & 8.8 \\
\hline Diabetes (\%) & & 1.5 & & 1.5 & & 1.5 & & 0.9 & & 1.7 & & 1.9 \\
\hline $\begin{array}{l}\text { Prevalent myocardial } \\
\text { infarction }(\%)\end{array}$ & & $1 \cdot 3$ & & 1.4 & & $1 \cdot 0$ & & $1 \cdot 0$ & & 1.5 & & 1.5 \\
\hline $\begin{array}{l}\text { Habitual food consumption } \\
\text { (servings/d) }\end{array}$ & & & & & & & & & & & & \\
\hline Total dairy products & 4.01 & $1 \cdot 87-7 \cdot 19$ & $3 \cdot 21$ & $1 \cdot 12-6 \cdot 30$ & 3.52 & $1.57-6.58$ & 3.81 & $1.94-7.08$ & 4.07 & $2 \cdot 40-6 \cdot 81$ & $5 \cdot 26$ & $3.39-8.49$ \\
\hline Low-fat milk & 0.46 & $0.05-1.71$ & 0.05 & $0.00-0.10$ & 0.19 & $0.13-0.27$ & 0.46 & $0.33-0.66$ & 0.88 & $0.77-1.12$ & 1.71 & $1.38-3.02$ \\
\hline Whole-fat milk & 0.15 & $0.01-0.59$ & 0.01 & $0.00-0.10$ & 0.06 & $0.02-0.55$ & 0.13 & $0.06-1 \cdot 10$ & 0.19 & $0.15-0.38$ & 0.37 & $0.27-0.72$ \\
\hline Buttermilk & 0.00 & $0.00-1.00$ & 0.00 & $0.00-2 \cdot 00$ & 0.00 & $0.00-2 \cdot 00$ & 0.00 & $0.00-1.00$ & 0.00 & $0.00-1.00$ & 0.00 & $0.00-1.00$ \\
\hline Low-fat yogurt & 0.15 & $0.01-0.68$ & 0.09 & $0.00-0.63$ & 0.15 & $0.01-0.74$ & 0.14 & $0.02-0.65$ & 0.17 & $0.02-0.68$ & 0.17 & $0.02-0.65$ \\
\hline Whole-fat yogurt & 0.05 & $0.00-0.28$ & 0.03 & $0.00-0.23$ & 0.05 & $0.01-0.29$ & 0.06 & $0.01-0.27$ & 0.06 & $0.01-0.29$ & 0.07 & $0.01-0.30$ \\
\hline Cheese & 1.50 & $0.36-3.38$ & 1.51 & $0.30-3.57$ & 1.50 & $0.34-3.39$ & 1.47 & $0.36-3.30$ & 1.50 & $0.43-3.25$ & 1.50 & $0.41-3.42$ \\
\hline Butter & 0.46 & $0.12-2.06$ & 0.40 & $0.08-2.08$ & 0.48 & $0.13-2 \cdot 26$ & 0.53 & $0.15-2.34$ & 0.44 & $0.12-1.77$ & 0.44 & $0.12-1.74$ \\
\hline Fruit & $2 \cdot 41$ & $0.77-4.77$ & $2 \cdot 39$ & $0.67-4.92$ & $2 \cdot 36$ & $0.69-4.75$ & $2 \cdot 20$ & $0.73-4.53$ & 2.48 & $0.92-4.73$ & 2.53 & $0.88-4.87$ \\
\hline Vegetables & 1.09 & $0.64-1.74$ & $1 \cdot 10$ & $0.61-1.82$ & 1.07 & $0.63-1.72$ & 1.07 & $0.63-1.70$ & 1.09 & $0.66-1.72$ & $1 \cdot 10$ & $0.63-1.74$ \\
\hline Fresh red meat & 0.41 & $0.11-0.70$ & 0.39 & $0.09-0.70$ & 0.41 & $0.11-0.71$ & 0.43 & $0.12-0.72$ & 0.40 & $0.12-0.67$ & 0.40 & $0.12-0.69$ \\
\hline Processed red meat & 1.37 & $0.20-4.07$ & 1.24 & $0.11-4.05$ & 1.36 & $0 \cdot 18-4 \cdot 10$ & 1.50 & $0.26-4.25$ & 1.34 & $0.23-3.94$ & 1.43 & $0 \cdot 23-4 \cdot 07$ \\
\hline Fish & 0.05 & $0.00-0.13$ & 0.06 & $0.00-0.13$ & 0.05 & $0.00-0.13$ & 0.05 & $0.00-0.13$ & 0.06 & $0.01-0.13$ & 0.06 & $0.00-0.13$ \\
\hline
\end{tabular}


Table 2. Associations for one serving/d substitutions between dairy products and risk of ischaemic stroke in the European Investigation into Cancer and Nutrition-Netherlands (EPIC-NL) cohort*

(Hazard ratios (HR) and $95 \%$ confidence intervals)

\begin{tabular}{|c|c|c|c|c|c|c|c|c|}
\hline \multirow[b]{2}{*}{ Substitution } & \multicolumn{2}{|c|}{ Model 1} & \multicolumn{2}{|c|}{ Model 2} & \multicolumn{2}{|c|}{ Model 3} & \multicolumn{2}{|c|}{ Model 4} \\
\hline & HR & $95 \% \mathrm{Cl}$ & HR & $95 \% \mathrm{Cl}$ & $\mathrm{HR}$ & $95 \% \mathrm{Cl}$ & HR & $95 \% \mathrm{Cl}$ \\
\hline \multicolumn{9}{|l|}{ Low-fat yogurt for } \\
\hline Whole-fat yogurt & 3.73 & $1.58,8 \cdot 81$ & $2 \cdot 74$ & $1 \cdot 18,6 \cdot 35$ & 2.58 & $1 \cdot 11,5 \cdot 97$ & $2 \cdot 27$ & $0.99,5.22$ \\
\hline Low-fat milk & 0.82 & $0.62,1.08$ & 0.86 & $0.66,1.13$ & 0.87 & $0 \cdot 67,1 \cdot 14$ & 0.87 & $0.67,1 \cdot 15$ \\
\hline Whole-fat milk & 0.72 & $0.52,1.00$ & 0.83 & $0.60,1.15$ & 0.85 & $0.61,1 \cdot 17$ & 0.80 & $0.58,1.11$ \\
\hline Buttermilk & 0.86 & $0.64,1.14$ & 0.90 & $0.68,1.18$ & 0.90 & $0.68,1.19$ & 0.89 & $0.68,1.18$ \\
\hline Cheese & 0.85 & $0 \cdot 65,1 \cdot 10$ & 0.89 & $0.70,1.15$ & 0.91 & $0.71,1 \cdot 17$ & 0.88 & $0.69,1.14$ \\
\hline Butter & 0.84 & $0.65,1.08$ & 0.91 & $0.71,1 \cdot 17$ & 0.93 & $0.72,1.19$ & 0.88 & $0.69,1.13$ \\
\hline \multicolumn{9}{|l|}{ Whole-fat yogurt for } \\
\hline Low-fat milk & 0.22 & $0.10,0.49$ & 0.32 & $0.14,0.70$ & 0.34 & $0.15,0.75$ & 0.38 & $0.18,0.85$ \\
\hline Whole-fat milk & 0.19 & $0.08,0.44$ & 0.30 & $0.13,0.69$ & 0.33 & $0.14,0.74$ & 0.35 & $0.16,0.79$ \\
\hline Buttermilk & 0.23 & $0.10,0.52$ & 0.33 & $0.15,0.73$ & 0.35 & $0.16,0.78$ & 0.39 & $0.18,0.87$ \\
\hline Cheese & 0.23 & $0.10,0.51$ & 0.33 & $0.15,0.71$ & 0.35 & $0.16,0.77$ & 0.39 & $0.18,0.85$ \\
\hline Butter & 0.22 & $0.10,0.50$ & 0.33 & $0.15,0.73$ & 0.36 & $0.16,0.79$ & 0.39 & $0.18,0.85$ \\
\hline \multicolumn{9}{|l|}{ Low-fat milk for } \\
\hline Whole-fat milk & 0.88 & $0.66,1 \cdot 17$ & 0.96 & $0.72,1.29$ & 0.97 & $0.73,1.30$ & 0.92 & $0.68,1.23$ \\
\hline Buttermilk & 1.05 & $0.89,1.24$ & 1.04 & $0.88,1.23$ & 1.03 & $0.88,1.22$ & 1.02 & $0.86,1.21$ \\
\hline Cheese & 1.04 & $0.90,1.20$ & 1.04 & $0.90,1.19$ & 1.04 & $0.90,1.20$ & 1.01 & $0.88,1.17$ \\
\hline Butter & 1.02 & $0.89,1.17$ & 1.05 & $0.92,1.21$ & 1.06 & $0.92,1.22$ & 1.01 & $0.88,1.16$ \\
\hline \multicolumn{9}{|l|}{ Whole-fat milk for } \\
\hline Buttermilk & 1.19 & $0.92,1.54$ & 1.08 & $0.83,1.39$ & 1.06 & $0 \cdot 82,1 \cdot 38$ & 1.12 & $0.86,1.44$ \\
\hline Cheese & $1 \cdot 18$ & $0.93,1.50$ & 1.08 & $0.85,1.37$ & 1.07 & $0.84,1.36$ & 1.11 & $0.87,1.40$ \\
\hline Butter & $1 \cdot 16$ & $0.90,1.49$ & 1.09 & $0.85,1.41$ & 1.09 & $0.85,1.41$ & 1.10 & $0.86,1.42$ \\
\hline \multicolumn{9}{|l|}{ Buttermilk for } \\
\hline Cheese & 0.99 & $0.85,1.15$ & 1.00 & $0.86,1.15$ & 1.01 & $0.87,1.16$ & 0.99 & $0.86,1.15$ \\
\hline Butter & 0.98 & $0.84,1 \cdot 13$ & 1.01 & $0.87,1 \cdot 18$ & 1.03 & $0.88,1.19$ & 0.99 & $0.85,1.15$ \\
\hline \multicolumn{9}{|l|}{ Cheese for } \\
\hline Butter & 0.98 & $0.88,1 \cdot 10$ & 1.02 & $0.91,1.13$ & 1.02 & $0.91,1.14$ & 1.00 & $0.89,1 \cdot 11$ \\
\hline
\end{tabular}

* Model 1 was adjusted for energy intake and cohort (stratum variable). Model 2 was additionally adjusted for education, BMI-adjusted waist circumference, smoking, physical activity and alcohol. Model 3 was additionally adjusted for the Dutch Health Diet Index 2015. Model 4 was additionally adjusted for baseline hypertension, hypercholesterolaemia, diabetes mellitus and myocardial infarction.

measure habitual diet in large-scale studies, the use of only one measurement may yield less precise risk estimates because the participants may have changed their diet during follow-up. The stroke diagnoses were identified by register linkage ${ }^{(14)}$ but were not individually validated; thus, non-differential misclassification of the outcome is possible ${ }^{(19)}$.

Despite elaborate model adjustment, residual confounding from unknown or unmeasured stroke risk factors and misclassification of covariates may still be present. However, given the magnitude of the associations after adjustment for lifestyle and dietary risk factors, residual confounding is unlikely to fully explain the inverse association of whole-fat yogurt as a substitution for other dairy products.

One other study has investigated substitutions between dairy product subgroups with the rate of stroke ${ }^{(11)}$. That study suggested that whole-fat yogurt substituted for low-fat yogurt, cheese, buttermilk or milk, regardless of fat content, was associated with a lower rate of ischaemic stroke, while no associations were observed for haemorrhagic stroke, which is in line with the results from the present study. The lack of association for substitutions between dairy product subgroups with haemorrhagic stroke could be due to a different aetiology of haemorrhagic compared with ischaemic stroke. However, both studies are likely underpowered to detect small associations, and therefore, strong conclusions from those analyses cannot be drawn.
Several prospective studies investigating dairy product intake and risk of stroke have been published ${ }^{(4-8,20,21)}$. These studies, however, did not specify a food substitution and, therefore, investigated the association of intake of dairy products in place of other non-specified energy-providing foods. Because what you choose to eat in place of a given dairy product may be population-specific and may affect the association, the studies are not readily comparable, and they do not directly address whether one type of dairy product is to be preferred over another.

Our results suggest that whole-fat yogurt may be a better alternative to other dairy products for the prevention of ischaemic stroke. In a randomised crossover study that compared the regular DASH diet with a modified high-fat DASH diet, including whole-fat dairy products rather than low-fat dairy products, similar beneficial effects on blood pressure were observed for both diets as well as favourable effects on the lipid profile with the high-fat DASH diet ${ }^{(3)}$. The compared diets had similar total energy contents but differed in the energy content provided by dairy foods. Consequently, the diets also differed in other energy-providing foods, in this case, particularly fruit juices and sugar from sweets. Therefore, the observed effects cannot solely be attributed to whole-fat dairy products because it is a joint effect of a higher intake of whole-fat dairy products and lower intake of fruit juices and sugars. In our study, the observed 
Table 3. Associations for one serving/d substitutions between dairy products and risk of haemorrhagic stroke in the European Investigation into Cancer and Nutrition-Netherlands (EPIC-NL) cohort*

(Hazard ratios (HR) and $95 \%$ confidence intervals)

\begin{tabular}{|c|c|c|c|c|c|c|c|c|}
\hline \multirow[b]{2}{*}{ Substitution } & \multicolumn{2}{|c|}{ Model 1} & \multicolumn{2}{|c|}{ Model 2} & \multicolumn{2}{|c|}{ Model 3} & \multicolumn{2}{|c|}{ Model 4} \\
\hline & $\mathrm{HR}$ & $95 \% \mathrm{Cl}$ & HR & $95 \% \mathrm{Cl}$ & $\mathrm{HR}$ & $95 \% \mathrm{Cl}$ & $\mathrm{HR}$ & $95 \% \mathrm{Cl}$ \\
\hline \multicolumn{9}{|l|}{ Low-fat yogurt for } \\
\hline Whole-fat yogurt & 1.06 & $0.37,3.02$ & 0.82 & $0.29,2.28$ & 0.75 & $0.27,2.09$ & 0.70 & $0.25,1.93$ \\
\hline Low-fat milk & 0.88 & $0.60,1.28$ & 0.90 & $0.62,1.30$ & 0.91 & $0.63,1.31$ & 0.90 & $0.63,1.30$ \\
\hline Whole-fat milk & 1.15 & $0.68,1.93$ & $1 \cdot 28$ & $0 \cdot 76,2 \cdot 16$ & $1 \cdot 30$ & $0.77,2.20$ & 1.25 & $0.74,2.11$ \\
\hline Buttermilk & 1.01 & $0.69,1.49$ & 1.02 & $0.70,1.49$ & 1.02 & $0.71,1.49$ & 1.02 & $0.70,1.47$ \\
\hline Cheese & 0.92 & $0.65,1.29$ & 0.95 & $0.68,1.32$ & 0.96 & $0.69,1.34$ & 0.95 & $0.68,1.32$ \\
\hline Butter & 0.84 & $0.60,1.17$ & 0.89 & $0.64,1.23$ & 0.91 & $0.66,1.25$ & 0.88 & $0.64,1.22$ \\
\hline \multicolumn{9}{|l|}{ Whole-fat yogurt for } \\
\hline Low-fat milk & 0.83 & $0.31,2 \cdot 21$ & $1 \cdot 10$ & $0.42,2.89$ & $1 \cdot 21$ & $0.46,3.17$ & 1.30 & $0.50,3.37$ \\
\hline Whole-fat milk & 1.08 & $0.37,3.12$ & 1.57 & $0.55,4.48$ & 1.74 & $0.61,4.96$ & 1.79 & $0.63,5.08$ \\
\hline Buttermilk & 0.95 & $0.35,2.57$ & 1.25 & $0.47,3.32$ & 1.36 & $0.52,3.60$ & 1.46 & $0.56,3.82$ \\
\hline Cheese & 0.87 & $0.33,2.27$ & $1 \cdot 16$ & $0.45,2.99$ & $1 \cdot 28$ & $0.50,3.30$ & 1.36 & $0.53,3.47$ \\
\hline Butter & 0.79 & $0.30,2.07$ & 1.09 & $0.42,2.81$ & 1.21 & $0.47,3.12$ & 1.27 & $0.50,3.24$ \\
\hline \multicolumn{9}{|l|}{ Low-fat milk for } \\
\hline Whole-fat milk & 1.31 & $0.78,2.20$ & 1.42 & $0.84,2.41$ & 1.44 & $0.85,2.43$ & 1.38 & $0.82,2.33$ \\
\hline Buttermilk & $1 \cdot 15$ & $0.90,1.48$ & $1 \cdot 14$ & $0.89,1.46$ & $1 \cdot 13$ & $0.88,1.44$ & $1 \cdot 12$ & $0.88,1.44$ \\
\hline Cheese & 1.05 & $0.85,1.28$ & 1.05 & $0.86,1.29$ & 1.06 & $0.87,1.29$ & 1.05 & $0.85,1.28$ \\
\hline Butter & 0.96 & $0.79,1.16$ & 0.99 & $0.82,1.20$ & 1.00 & $0.83,1.21$ & 0.98 & $0.81,1.19$ \\
\hline \multicolumn{9}{|l|}{ Whole-fat milk for } \\
\hline Buttermilk & 0.88 & $0.56,1.39$ & 0.80 & $0.50,1.27$ & 0.79 & $0.50,1.25$ & 0.81 & $0.51,1.29$ \\
\hline Cheese & 0.80 & $0.52,1.23$ & 0.74 & $0.48,1.15$ & 0.74 & $0.48,1.14$ & 0.76 & $0.49,1.17$ \\
\hline Butter & 0.73 & $0.47,1.14$ & 0.70 & $0.44,1.10$ & 0.70 & $0.44,1 \cdot 10$ & 0.71 & $0.45,1.11$ \\
\hline \multicolumn{9}{|l|}{ Buttermilk for } \\
\hline Cheese & 0.91 & $0.73,1.13$ & 0.93 & $0.75,1.14$ & 0.94 & $0.76,1 \cdot 16$ & 0.93 & $0.76,1.15$ \\
\hline Butter & 0.83 & $0.67,1.02$ & 0.87 & $0.71,1.07$ & 0.89 & $0.72,1.09$ & 0.87 & $0.71,1.07$ \\
\hline \multicolumn{9}{|l|}{ Cheese for } \\
\hline Butter & 0.91 & $0.80,1.05$ & 0.94 & $0.82,1.08$ & 0.95 & $0.82,1.09$ & 0.93 & $0.81,1.07$ \\
\hline
\end{tabular}

* Model 1 was adjusted for energy intake and cohort (stratum variable). Model 2 was additionally adjusted for education, BMI-adjusted waist circumference, smoking, physical activity and alcohol. Model 3 was additionally adjusted for the Dutch Health Diet Index 2015. Model 4 was additionally adjusted for baseline hypertension, hypercholesterolaemia, diabetes mellitus and myocardial infarction.

associations also represent a joint association, namely, the comparison of two specified dairy subgroups. However, when we compare equal serving sizes of products differing in energy content or foods with different serving sizes, a difference in energy from other non-specified foods will remain unexplained by the model, but this unexplained residual energy is much smaller than if we had not specified substitutions. In order to address the influence of unexplained residual energy intake in our analyses, we also performed isoenergetic substitution analyses of 100 $\mathrm{kcal} / \mathrm{d}(418 \mathrm{~kJ} / \mathrm{d})$, which roughly corresponds to the energy content of a glass of low-fat milk. The results from these analyses did not markedly differ from the results based on servings, which implies that unexplained energy intake may not be a major issue in the analyses of servings. This is perhaps because the difference in energy content of equal servings of milk and yogurt is relatively small in the light of the total daily energy intake. Also, despite a large difference in energy density between milk products and cheese and butter, this difference is compensated for by the large difference in serving size. Consequently, in the case of substitution of dairy products, the two analytical approaches are comparable. This may, however, not always be the case. For instance $100 \mathrm{kcal} / \mathrm{d}(418 \mathrm{~kJ} / \mathrm{d})$ isoenergetic substitutions between spinach and regular-fat cheese will approximately compare $400 \mathrm{~g}$ of spinach with $30 \mathrm{~g}$ of cheese. While such an analysis is not confounded by a difference in energy intake of other foods, it does not reflect the choices of substitution made in the population. Thus, both analytical approaches hold important information but have limitations.

Multiple mechanisms might explain why whole-fat yogurt products appear to be a better alternative than other dairy products. Dairy fat contains conjugated linoleic acid, which has been proposed to have beneficial health effects related to atherosclerosis, such as changes in body fat, lipid profile and blood pressure ${ }^{(22,23)}$. Conjugated linoleic acid is formed in the rumen and mammary gland of the cow and, to a lesser extent, endogenously in humans by the conversion of vaccenic acid in dairy fat ${ }^{(24-26)}$. Fermented milk products, such as yogurt, contain probiotic cultures, such as lactobacilli and bifidobacteria, that may further increase the content of conjugated linoleic acid through the conversion of linoleic $\operatorname{acid}^{(27)}$. In addition, different probiotic cultures may also individually exert anti-atherosclerotic effects ${ }^{(23)}$. Therefore, our results may reflect a synergistic effect of high fat content and the presence of probiotic bacteria. The fat content alone does not appear to explain the found associations, as we did not find an association for the substitution between low-fat and whole-fat milk. Similarly, the presence of probiotic bacteria alone does not appear to explain the found associations, as we did not find an association for the substitution of low-fat yogurt for low-fat milk. Furthermore, milk intake has been positively associated with urinary 8-iso-PGF2 $\alpha$, a biomarker of oxidative stress, 
whereas fermented milk intake has been negatively associated with 8-iso-PGF2 $\alpha^{(28)}$, offering an additional possible explanation for why whole-fat yogurt products as a substitution for milk are associated with a lower rate of ischaemic stroke in our study.

In conclusion, we observed that the substitution of whole-fat yogurt products for low-fat yogurt products, cheese, butter, buttermilk or milk, regardless of fat content, was associated with a lower rate of ischaemic but not haemorrhagic stroke.

\section{Supplementary material}

For supplementary material/s referred to in this article, please visit https://doi.org/10.1017/S0007114519000564

\section{Acknowledgements}

The EPIC-NL study was supported by the 'Europe Against Cancer' Programme of the European Commission; Dutch Ministry of Health, Welfare and Sports; Netherlands Organisation for Health Research and Development; and World Cancer Research Fund. I. S. reports grants from Unilever Research and Development, Vlaardingen, The Netherlands.

A. S. D. L. and M. U. J. designed the study; A. S. D. L. analysed the data; A. S. D. L., M. U. J., I. S. and Y. v. d. S. interpreted the data. A. S. D. L. wrote the manuscript. J. M. A. B. and W. M. M. V. provided input to the manuscript. All authors read and approved the final version of the manuscript.

The authors declare that there are no conflicts of interest.

\section{References}

1. Appel LJ, Moore TJ, Obarzanek E, et al. (1997) A clinical trial of the effects of dietary patterns on blood pressure. DASH Collaborative Research Group. N Engl J Med 336, 1117-1124.

2. Goldstein LB, Bushnell CD, Adams RJ, et al. (2011) Guidelines for the primary prevention of stroke: a guideline for healthcare professionals from the American Heart Association/American Stroke Association. Stroke 42, 517-584.

3. Chiu S, Bergeron N, Williams PT, et al. (2016) Comparison of the DASH (Dietary Approaches to Stop Hypertension) diet and a higher-fat DASH diet on blood pressure and lipids and lipoproteins: a randomized controlled trial. Am J Clin Nutr 103, 341-347.

4. Louie JC, Flood VM, Burlutsky G, et al. (2013) Dairy consumption and the risk of 15 -year cardiovascular disease mortality in a cohort of older Australians. Nutrients 5, 441-454.

5. Larsson SC, Virtamo J \& Wolk A (2012) Dairy consumption and risk of stroke in Swedish women and men. Stroke 43, 17751780 .

6. Sonestedt E, Wirfalt E, Wallstrom P, et al. (2011) Dairy products and its association with incidence of cardiovascular disease: the Malmo diet and cancer cohort. Eur J Epidemiol 26, 609-618.

7. Dalmeijer GW, Struijk EA, van der Schouw YT, et al. (2013) Dairy intake and coronary heart disease or stroke - a population-based cohort study. Int J Cardiol 167, 925-929.

8. Praagman J, Franco OH, Ikram MA, et al. (2015) Dairy products and the risk of stroke and coronary heart disease: the Rotterdam Study. Eur J Nutr 54, 981-990.
9. Gaucheron F (2011) Milk and dairy products: a unique micronutrient combination. J Am Coll Nutr 30, 400s-409s.

10. de Goede J, Soedamah-Muthu SS, Pan A, et al. (2016) Dairy consumption and risk of stroke: a systematic review and updated dose-response meta-analysis of prospective cohort studies. J Am Heart Assoc 5, e002787.

11. Laursen ASD, Dahm CC, Johnsen SP, et al. (2017) Substitutions of dairy product intake and risk of stroke: a Danish cohort study. Eur J Epidemiol 33, 201-212.

12. Beulens JWJ, Monninkhof EM, Verschuren WMM, et al. (2010) Cohort profile: the EPIC-NL study. In J Epidemiol 39, $1170-1178$.

13. Ocke MC, Bueno-de-Mesquita HB, Goddijn HE, et al. (1997) The Dutch EPIC food frequency questionnaire. I. Description of the questionnaire, and relative validity and reproducibility for food groups. Int J Epidemiol 26, Suppl.1, S37-S48.

14. Herings RM, Bakker A, Stricker BH, et al. (1992) Pharmacomorbidity linkage: a feasibility study comparing morbidity in two pharmacy based exposure cohorts. J Epidemiol Commun Health 46, 136-140.

15. Wareham NJ, Jakes RW, Rennie KL, et al. (2003) Validity and repeatability of a simple index derived from the short physical activity questionnaire used in the European Prospective Investigation into Cancer and Nutrition (EPIC) study. Public Health Nutr 6, 407-413.

16. Sluijs I, van der AD, Beulens JW, et al. (2010) Ascertainment and verification of diabetes in the EPIC-NL study. Neth J Med $\mathbf{6 8}$, 333-339.

17. Looman M, Feskens EJ, de Rijk M, et al. (2017) Development and evaluation of the Dutch Healthy Diet index 2015. Public Health Nutr 20, 2289-2299.

18. Kromhout D, Spaaij CJ, de Goede J, et al. (2016) The 2015 Dutch food-based dietary guidelines. Eur J Clin Nutr 70, 869-878.

19. McCormick N, Bhole V, Lacaille D, et al. (2015) Validity of diagnostic codes for acute stroke in administrative databases: a systematic review. PLOS ONE 10, e0135834.

20. Goldbohm RA, Chorus AM, Galindo Garre F, et al. (2011) Dairy consumption and 10-y total and cardiovascular mortality: a prospective cohort study in the Netherlands. Am J Clin Nutr $\mathbf{9 3}$, 615-627.

21. Larsson SC, Mannisto S, Virtanen MJ, et al. (2009) Dairy foods and risk of stroke. Epidemiology 20, 355-360.

22. Dilzer A \& Park Y (2012) Implication of conjugated linoleic acid (CLA) in human health. Crit Rev Food Sci Nutr 52, 488-513.

23. DiRienzo DB (2014) Effect of probiotics on biomarkers of cardiovascular disease: implications for heart-healthy diets. Nutr Rev 72, 18-29.

24. Collomb M, Schmid A, Sieber R, et al. (2006) Conjugated linoleic acids in milk fat: variation and physiological effects. Int Dairy J 16, 1347-1361.

25. Jakobsen MU, Bysted A, Andersen NL, et al. (2006) Intake of ruminant trans fatty acids in the Danish population aged 1-80 years. Eur J Clin Nutr 60, 312-318.

26. Turpeinen AM, Mutanen M, Aro A, et al. (2002) Bioconversion of vaccenic acid to conjugated linoleic acid in humans. $A m J$ Clin Nutr 76, 504-510.

27. Andrade JC, Ascenção K, Gullón P, et al. (2012) Production of conjugated linoleic acid by food-grade bacteria: a review. Int $J$ Dairy Technol 65, 467-481.

28. Michaëlsson K, Wolk A, Langenskiöld S, et al. (2014) Milk intake and risk of mortality and fractures in women and men: cohort studies. BMJ 349, g6015. 